\title{
Efficient Calculation of Non-Orthogonal Partial Elements for the PEEC Method
}

\author{
A. Müsing ${ }^{1}$, J. Ekman ${ }^{2}$, and J. W. Kolar ${ }^{1}$ \\ ${ }^{1}$ Power Electronic Systems Laboratory, ETH Zürich, CH-8092 Zürich, Switzerland \\ ${ }^{2}$ EISLAB, Luleå University of Technology, SE-971 87 Luleå, Sweden
}

\begin{abstract}
For various electrical interconnect and EMC problems, the partial element equivalent circuit (PEEC) method has proven to be a valid and fast solution method of the electrical field integral equation in the time as well as the frequency domain. Therefore, PEEC has become a multipurpose full-wave method, especially suited for the solution of combined circuit and EM problems, as found, for instance, on printed circuit board layouts, power electronics devices or EMC filters. Recent research introduced various extensions to the basic PEEC approach, for example a non-orthogonal cell geometry formulation. This paper presents a fast, flexible and accurate computational method for determining the matrix entries of partial inductances and the coefficients of potential for general non-orthogonal PEEC cell geometries. The presented computation method utilizes analytical filament formulas to reduce the integration order and therefore to reduce computation time. The validity, accuracy, and speed of the proposed method is compared with a standard integration routine on example cell geometries where the numeric results of the new method show improved accuracy, coming along with reduced computation time.
\end{abstract}

Index Terms-Filament mutual inductance, integration, non-orthogonal partial element equivalent circuit (PEEC), partial element computation.

\section{INTRODUCTION}

$\mathbf{T}$ HE increasing performance of electronic circuits occurs by increasing the complexity and the operating frequencies. This fact is true for on-chip VLSI design, as well as for macroscopic circuits as in power electronics. Due to the resulting fast voltage and current transients, the modeling of electric interconnects and the analysis of their electromagnetic behavior is gaining in importance.

For the solution of mixed EM problems, the partial element equivalent circuit (PEEC) method [1] has become a very popular approach. The method is based on a circuit interpretation of the electric field integral equation (EFIE). Unlike the method of moments, PEEC is a full-spectrum method valid from dc to a maximum frequency determined by the meshing. Further extensions, especially the introduction of time retardation [2], dielectric cells [3] and the formulation with nonorthogonal cell geometries [4] have made PEEC a multipurpose electromagnetic solver.

A PEEC simulation is mainly performed in the following steps.

- geometry discretization of the layout and placement of external components such as current and voltages sources or load impedances;

- fill-in of partial inductance matrices $L_{p}$ and partial coefficients of potential matrices $P_{n}$;

- setup of the matrix equation system, typically in modified nodal analysis (MNA) formulation;

- time domain or frequency domain solution of the system matrix equation in a SPICE-like solver.

Manuscript received October 07, 2008. Current version published February 19, 2009. Corresponding author: A. Müsing (e-mail: muesing@lem.ee.ethz.ch). Color versions of one or more of the figures in this paper are available online at http://ieeexplore.ieee.org.

Digital Object Identifier 10.1109/TMAG.2009.2012655
The bottlenecks of simulation effort are both, matrix fill-in and the subsequent solution of the equation systems, which limit the maximum problem size to $10^{4}-10^{5}$ unknown variables, namely the node voltages and currents. The partial element computation is the limiting factor in particular for non-orthogonal cell geometries, since fast analytical formulas are not directly applicable. Hence, a cumbersome multidimensional integration must be performed numerically for every entry in the $L_{p}$ and $P_{n}$ matrices. Furthermore, the obtained accuracy of partial elements has direct influence on the stability of time-domain models [5] and a fast computation with high precision is desirable.

This paper is introducing a new integration method using analytic solutions for the Neumann integral along straight and arbitrarily aligned current filaments [6] to accelerate the calculation of non-orthogonal partial elements. This technique can be applied to the mutual couplings between the PEEC cells as well as the self-terms represented by the diagonal elements of the $L_{p}$ and $P_{n}$ matrices.

\section{NON-ORTHOGONAL PEEC FORMULATION}

The classical PEEC method is derived from the equation for the total electric field at a point written as

$$
\boldsymbol{E}_{i}(\boldsymbol{r}, t)=\frac{\boldsymbol{J}(\boldsymbol{r}, t)}{\sigma}+\frac{\partial \boldsymbol{A}(\boldsymbol{r}, t)}{\partial t}+\nabla \phi(\boldsymbol{r}, t)
$$

where $\boldsymbol{E}_{i}$ is an incident electric field, $\boldsymbol{J}$ is a current density, $\boldsymbol{A}$ is the magnetic vector potential, and $\phi$ is the scalar electric potential. By using the definitions of the scalar and vector potentials, the current- and charge densities are discretized by defining pulse basis functions for the conductors and dielectric materials. Pulse functions are also used for the weighting functions resulting in a Galerkin type solution. By defining a suitable inner product, a weighted volume integral over the cells, the field (1) can be interpreted as Kirchhoff's voltage law over a PEEC cell consisting of partial self inductances between the nodes and partial mutual inductances representing the magnetic 
(a)

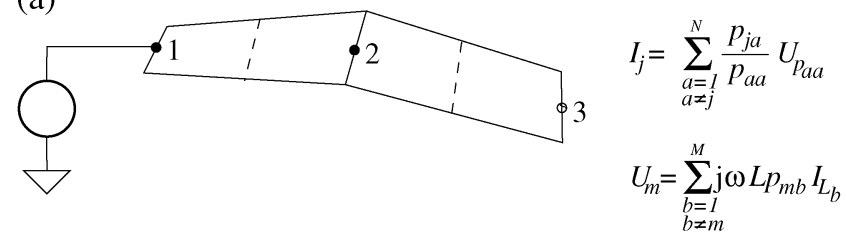

(b)

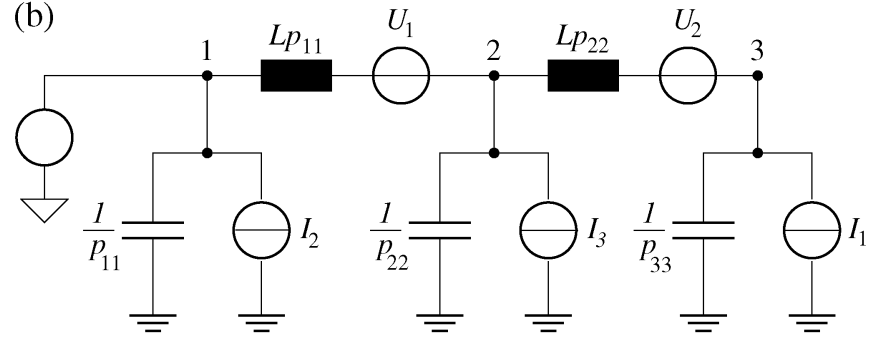

Fig. 1. (a) Two non-orthogonal conductor cells and (b) their corresponding equivalent circuit.

field coupling in the equivalent circuit. The partial inductances, shown as $L p_{i j}$ in Fig. 1, are defined as

$$
L p_{i j}=\frac{\mu}{4 \pi} \frac{1}{a_{i} a_{j}} \iint_{v_{i} v_{j}} \frac{1}{\left|\boldsymbol{r}_{i}-\boldsymbol{r}_{j}\right|} \mathrm{d} v_{i} \mathrm{~d} v_{j} .
$$

Fig. 1 also shows the node capacitances which are related to the diagonal coefficients of potential $p_{i i}$ while ratios consisting of $p_{i j} / p_{i i}$ are leading to the current sources in the PEEC circuit. The coefficients of potentials are computed as

$$
p_{i j}=\frac{1}{4 \pi \epsilon_{0}} \frac{1}{S_{i} S_{j}} \iint_{S_{i} S_{j}} \frac{1}{\left|\boldsymbol{r}_{i}-\boldsymbol{r}_{j}\right|} \mathrm{d} S_{i} \mathrm{~d} S_{j} .
$$

Introducing a local non-orthogonal coordinate system $\left(a, b, c, a^{\prime}, b^{\prime}, c^{\prime}\right)$, the general self and mutual partial inductances can be extended to a non-orthogonal formulation as detailed in [4]. Then, the inductances are computed as

$$
L p_{a a^{\prime}}=\mu \iiint_{a} \iiint_{a^{\prime} b^{\prime} c^{\prime}} \hat{\boldsymbol{a}}^{\prime} \cdot \hat{\boldsymbol{a}}\left|\frac{\partial \boldsymbol{r}}{\partial a}\right|\left|\frac{\partial \boldsymbol{r}^{\prime}}{\partial a^{\prime}}\right| G\left(\boldsymbol{r}, \boldsymbol{r}^{\prime}\right) \mathrm{d} v \mathrm{~d} v^{\prime}
$$

and likewise, the coefficients of potential are given by

$$
P n_{a a^{\prime}}=\frac{1}{\epsilon} \iiint_{a} \int_{a^{\prime} b^{\prime}} G\left(\boldsymbol{r}, \boldsymbol{r}^{\prime}\right) \mathrm{d} A \mathrm{~d} A^{\prime} .
$$

The double volume and double surface integrations in (4) and (5) are performed in cell coordinates of the corresponding hexahedral or quadrilateral PEEC cells and the free space Green's function is used

$$
G\left(\boldsymbol{r}, \boldsymbol{r}^{\prime}\right)=\frac{\mathrm{e}^{-\mathrm{j} k\left(r-r^{\prime}\right)}}{4 \pi\left|\boldsymbol{r}-\boldsymbol{r}^{\prime}\right|} \approx \frac{1}{4 \pi\left|\boldsymbol{r}-\boldsymbol{r}^{\prime}\right|}
$$

where, throughout this paper, the time retardation is neglected.

\section{Non-ORTHOgOnal Partial ElEMENT CALCUlation \\ Using ANALYTIC FILAMENT Formulas}

The numeric evaluation of (4) and (5) requires a huge computational effort due to the dimensional manifold and the fact

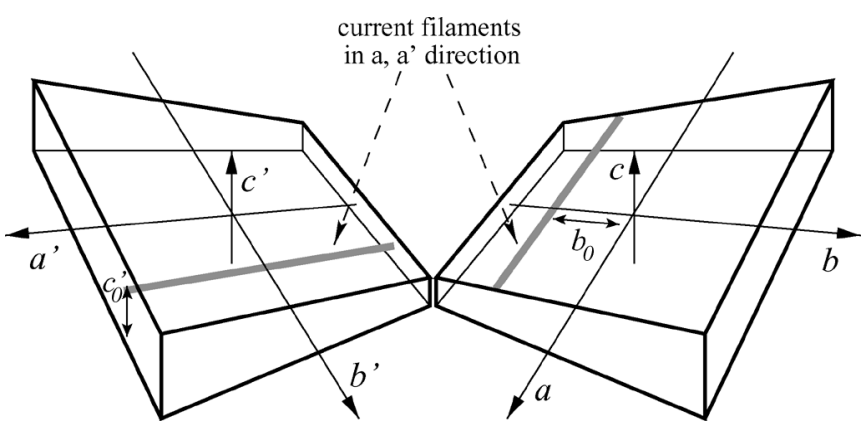

Fig. 2. Two inductive hexahedral PEEC volume cells with current direction $a$ and $a^{\prime}$. The coordinate axes indicate a local non-orthogonal coordinate system, as introduced in [4]. Furthermore, two current filaments, pointing respectively in $a$ and $a^{\prime}$ direction, are displayed.

that the $L p$ - and $P n$-matrices are dense and full, respectively. In particular, the self terms on the matrix diagonals require special attention due to the singularity in Green's function (6).

Insertion of a four-fold $\delta$-pulse function into the non-orthogonal $L p$ definition (4) gives a relation between the mutual inductance of infinitesimal thin current filaments and the non-orthogonal volume mutual inductance

$$
\begin{aligned}
L p_{a a^{\prime}}= & \mu \iint_{a} \int_{b} \int_{c} \int_{a^{\prime}} \int_{b^{\prime}} \delta\left(b-b_{0}, b^{\prime}-b_{0}^{\prime}, c-c_{0}, c^{\prime}-c_{0}^{\prime}\right) \cdots \\
& \hat{\boldsymbol{a}}^{\prime} \cdot \hat{\boldsymbol{a}}\left|\frac{\partial \boldsymbol{r}}{\partial a}\right|\left|\frac{\partial \boldsymbol{r}^{\prime}}{\partial a^{\prime}}\right| G\left(\boldsymbol{r}, \boldsymbol{r}^{\prime}\right) \mathrm{d} v \mathrm{~d} v^{\prime} \\
& =L p_{f f}\left(b_{0}, b_{0}^{\prime}, c_{0}, c_{0}^{\prime}\right) .
\end{aligned}
$$

The geometric proportions are exemplified in Fig. 2. Taking into account the integration property of Dirac's $\delta$ pulse function ${ }^{1}$ reduces the double volume integration (4) into a double surface integral. Therefore, one obtains a simplified expression for the partial mutual inductance between two non-orthogonal volume cells

$$
L p_{a a^{\prime}}=\iint_{b} \int_{b^{\prime}} \int_{c} \int_{c^{\prime}} L p_{f f}\left(b, b^{\prime}, c, c^{\prime}\right) \mathrm{d} a \mathrm{~d} a^{\prime} \mathrm{d} b \mathrm{~d} b^{\prime} .
$$

Hence, $L p$ in (4) can be expressed using only the integral of the analytic solution $L p_{f f}()$ for mutual inductances between arbitrary aligned current filaments, which are well-known [7] (see Appendix). The filament formula is a complex expression including several hyperbolic and trigonometric functions. Nevertheless, a numeric evaluation of (8) speeds up the integration. Besides the integration order reduction, the speedup is caused by a smoothing of the Green's function singularity (8), which is shown in Fig. 3. In particular, this smoothing property facilitates a fast calculation of the diagonal matrix entries, for which the volumes $v$ and $v^{\prime}$ in (4) coincide.

Similar to the shown procedure and with small modifications to the filament formulas, the partial coefficients of potential calculation (5) can be reduced to a double line integral which is possible due to the mathematical similarity between (4) and (5). The main differences in the $P n$ matrix calculation are the missing dot product and a constant surface charge density in (5), in contrast to a variable volume current density in (4). The

$$
{ }^{1} \int \delta\left(x-x_{0}\right) f(x) \mathrm{d} x=f\left(x_{0}\right) .
$$




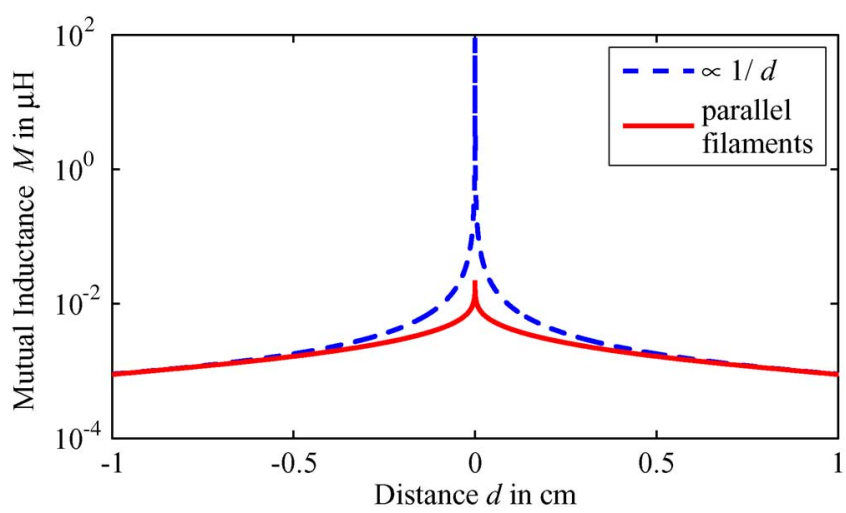

Fig. 3. Mutual inductance between two parallel current filaments of length $l=$ $1 \mathrm{~cm}$. Approaching a zero distance $d$, the inductance $M$ is diverging to infinity. However, the divergence $\propto \log (2 l / r)$ is relatively weak in comparison to the $1 / d$ Green's function, which allows a more efficient numeric integration of the partial elements.

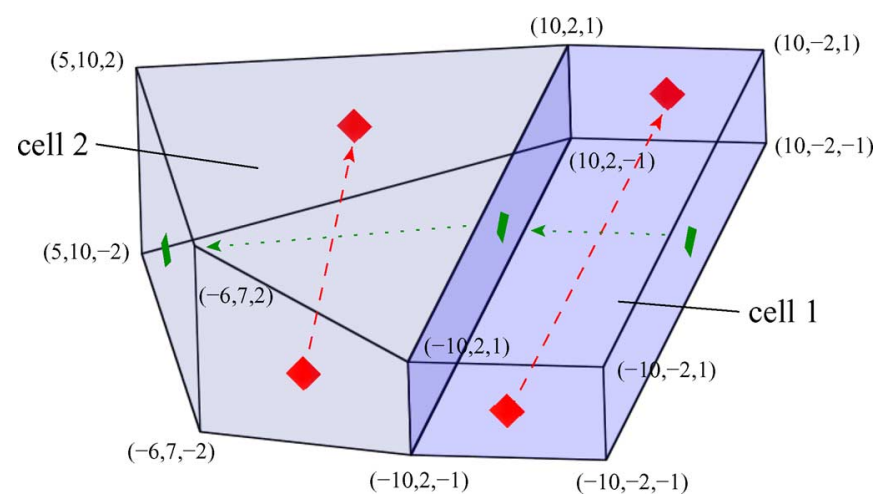

Fig. 4. Example cell geometry: two inductive PEEC cells. Two different current directions (dashed and dotted lines) and the cell corner point coordinates are given (in millimeters) so that numeric results are replicable.

remainder of this paper will focus on inductance calculations, keeping in mind that the same technique of accelerated matrix fill-in can be applied for the coefficients of potential, as well.

The proposed accelerated partial element calculation was implemented in $\mathrm{C}++$, using an adaptive integration routine based on a recursive Simpson algorithm with error estimation [8]. This gives the opportunity to select any desired integration accuracy. Furthermore, the adaptive algorithm selects autonomously the number of interpolation points, which lowers the number of filament evaluations for inductive cells that are far apart, and refines the integration for adjacent cells or the self-terms.

\section{Non-ORThOGONAL APPLICATION EXAMPLE}

Fig. 4 shows an example of two inductive PEEC cells, for which the $L p$ calculation is investigated in the following. Cell 1 was chosen to be orthogonal. Therefore, an accurate reference value of $L_{11}$ can be obtained from an analytic exact solution [9]. Cell 2 is a non-orthogonal hexahedral, hence for $L_{12}$ and $L_{22}$, a numeric integration is mandatory. The proximity of cell 1 and 2 is a worst case for the mutual inductance calculation. Here, the recursive Simpson algorithm will increase the number of filament evaluations near the contact surface. The aspect ratio (length/thickness $\approx 10$ ) of the shown cells is quite large. However, such thin and non-orthogonal structures are typical for
TABLE I

COMPARISON OF INTEGRATION METHODS

\begin{tabular}{|c|c|c|c|c|}
\hline & $L p_{11}[\mathrm{nH}]$ & $L p_{12}[\mathrm{nH}]$ & $L p_{12}[\mathrm{nH}]$ & $L p_{34}[\mathrm{nH}]$ \\
& $N$ & $N$ & $N$ & $N$ \\
& $T[\mathrm{msec}]$ & $T[\mathrm{msec}]$ & $T[\mathrm{msec}]$ & $T[\mathrm{msec}]$ \\
\hline exact & 9.8936 & - & - & - \\
\hline new filament & 9.8934 & 5.70895 & 4.52591 & 0.364282 \\
integration & $9 \mathrm{E} 6$ & $9 \mathrm{E} 6$ & $2 \mathrm{E} 5$ & $2 \mathrm{E} 5$ \\
algorithm & $1 \mathrm{E} 4$ & $1 \mathrm{E} 4$ & 320 & 340 \\
\hline & 9.8921 & 5.70881 & 4.5260 & 0.36438 \\
$* *$ & $1 \mathrm{E} 5$ & $1 \mathrm{E} 5$ & 14065 & 12889 \\
& 130 & 200 & 22 & 21 \\
\hline$*$ & 9.8958 & 5.71733 & 4.52618 & 0.369 \\
& 2401 & 2401 & 3277 & 2485 \\
& 2.7 & 3.9 & 5.2 & 4.1 \\
\hline conventional & 9.58759 & 5.424 & 4.16366 & 0.3633 \\
GL & $2 \mathrm{E} 6$ & $2 \mathrm{E} 6$ & $2 \mathrm{E} 6$ & $2 \mathrm{E} 6$ \\
integration & 2000 & 2000 & 2000 & 2000 \\
\hline & 9.4819 & 5.025 & 4.153 & 0.3625 \\
& $7 \mathrm{E} 5$ & $7 \mathrm{E} 5$ & $7 \mathrm{E} 5$ & $7 \mathrm{E} 5$ \\
& 600 & 600 & 600 & 600 \\
\hline
\end{tabular}

Inductance values $L p_{i j}$ of the non-orthogonal cell geometry from Fig. 2, obtained from different integration routines and with varying numeric integration resolutions. Additionally, the number of integration steps $N$ and the corresponding computation time $T$ is given for every inductance value.

many conductor geometries, e.g., [10] and [11]. To obtain another test case of mutual inductances, additionally to the main current direction in Fig. 4 (dashed line), a second direction was introduced (dotted line, corresponding $L p_{34}$ ).

The numeric inductance results are outlined in Table I for different integration resolutions. Here, the conventional Gauss-Legendre (GL) integration is directly applied to (4) and $N$ gives the number of GL evaluation points. In the case of the proposed filament algorithm using (8), $N$ is the total number of filament evaluations. Furthermore, the total computation time on a $3 \mathrm{GHz}$ $\mathrm{CPU}$ is given, respectively.

Due to the singularity in the Green's function (6), GL integration consequently underestimates inductances. Even with fine resolution and hence with big numeric effort, the results do not have a satisfactory accuracy. In contrast, the new proposed method shows a good convergence behavior, which results in a more reasonable computation time.

Obviously, the most time intensive computations are still performed for the matrix diagonal self-terms. Off-diagonal element computations need less computation time, which is further diminished with the increasing geometric cell distance. For realistic problem sizes with, e.g., $n=10^{4}$ non-orthogonal cells, the new method allows a $L_{p}$ and $P_{n}$ fill-in within $1 \mathrm{~h}$ for $* *$-accuracy in Table I, or about 10 min for $*$-accuracy, whereas GL integration would require orders of magnitudes more computation time.

\section{CONCLUSION AND OUTLOOK}

This paper proposed a new numeric method for the evaluation of general non-orthogonal partial element matrix entries for PEEC simulations. With the usage of analytic mutual inductance formulas between arbitrary aligned current filaments, accuracy and speed of the partial element calculation is improved by orders of magnitudes in comparison to a conventional 


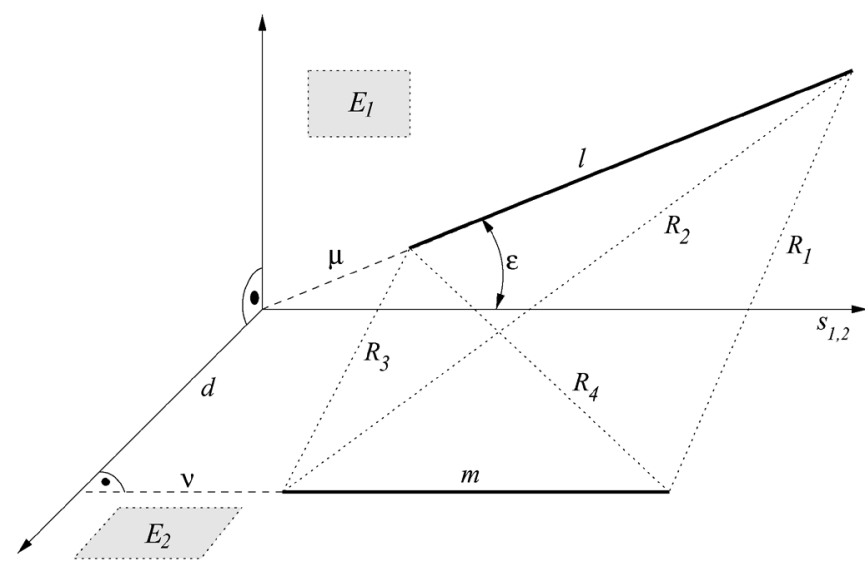

Fig. 5. Shown are two arbitrarily aligned current filaments $l$ and $m$ and the required distances and angles for the mutual inductance calculation, see equations (9) and (10). Two Planes $E_{1}$ and $E_{2}$ are passed through the filaments in such a way as to intersect at right angles. Here, $s_{1,2}$ is the intersection line between the planes, having the filament $m$ in parallel. The geometrical filament arrangement is completely determined by 6 independent parameters, e.g. $R_{1, \ldots, 4}, m$, and $l$.

Gauss-Legendre integration routine. This work focuses on the inductance calculations, whereas the same technique is applicable for the coefficients of potential.

An extension of the proposed method for high frequency applications could be to take into account time retardation. In principle, a derivation of analytic filament formulas in [6] can be performed for a complex valued Green's function (6), resulting in complex $L p$ and $P n$ entries. This could be used to further improve time domain stability in comparison to the common used center-to-center retardation approximation [2].

\section{APPENDIX}

Analytical solutions for the mutual inductance $M$ between two straight current filaments, placed in any desired position, are given in [6], [7], and [13]. Due to the old age of the citations and for the sake of completeness, the inductance formula is briefly recapitulated. Equations (9) and (10) show the expressions used for the partial element integration in this paper

$$
\begin{array}{r}
M=L p_{f f}\left(R_{1}, R_{2}, R_{3}, R_{4}\right)=\cos \varepsilon \iint \frac{\mathrm{d} x \mathrm{~d} y}{R} \\
=2 \cos \varepsilon\left[(\mu+l) \tanh ^{-1} \frac{m}{R_{1}+R_{2}}\right. \\
+(\nu+m) \tanh ^{-1} \frac{l}{R_{1}+R_{4}} \\
-\mu \tanh ^{-1} \frac{m}{R_{3}+R_{4}} \\
\left.-\nu \tanh ^{-1} \frac{l}{R_{2}+R_{3}}-\frac{\Omega d}{2 \tan \varepsilon}\right]
\end{array}
$$

$$
\text { with } \begin{aligned}
\Omega= & \tanh ^{-1} \frac{d^{2} \cos \varepsilon+(\mu+l)(\nu+m) \sin ^{2} \varepsilon}{d R_{1} \sin \varepsilon} \\
& -\tanh ^{-1} \frac{d^{2} \cos \varepsilon+(\mu+l) \nu \sin ^{2} \varepsilon}{d R_{2} \sin \varepsilon} \\
& +\tanh ^{-1} \frac{d^{2} \cos \varepsilon+\mu \nu \sin ^{2} \varepsilon}{d R_{3} \sin \varepsilon} \\
& -\tanh ^{-1} \frac{d^{2} \cos \varepsilon+\mu(\nu+m) \sin ^{2} \varepsilon}{d R_{4} \sin \varepsilon} .
\end{aligned}
$$

The required length and angle parameters can be extracted from Fig. 5. The inductance formula is analytically exact, however, care must be taken of the numerical evaluation. For special geometric arrangements, the hyperbolic functions as well as the fractions in (9) and (10) diverge to infinity. For instance, this is the case when the filaments intersect, touch or when they are in parallel. Nevertheless, the analytical limit is always well defined, and the use of a series expansion is required to obtain accurate results when such a divergence is detected.

\section{REFERENCES}

[1] A. E. Ruehli, "Equivalent circuit models for three dimensional multiconductor systems," IEEE Trans. Microw. Theory Techn., vol. 22, no. 3, pp. 216-221, Mar. 1974.

[2] H. Heeb and A. E. Ruehli, "Retarded models for PC board interconnects-Or how the speed of light affects your SPICE circuit simulation," in Proc. ICCAD, 1991, pp. 70-73.

[3] A. E. Ruehli and H. Heeb, "Circuit models for three-dimensional geometries including dielectrics," IEEE Trans. Microw. Theory Techn., vol. 40, no. 7, pp. 1507-1516, Jul. 1992.

[4] A. E. Ruehli, G. Antonini, J. Esch, J. Ekman, A. Mayo, and A. Orlandi, "Nonorthogonal PEEC formulation for time- and frequency-domain EM and circuit modeling," IEEE Trans. Electromagn. Compatibility, vol. 45, no. 2, pp. 167-176, May 2003.

[5] J. Ekman, G. Antonini, A. Orlandi, and A. E. Ruehli, "Impact of partial element accuracy on PEEC model stability," IEEE Trans. Electromagn. Compatibility, vol. 48, no. 1, pp. 19-31, Feb. 2006.

[6] F. F. Martens, "Über die gegenseitige Induktion und pondermotorische Kraft zwischen zwei stromdurchflossenen Rechtecken," Ann. der Phys., vol. 343, no. 10, pp. 959-970, 1909.

[7] F. W. Grover, Inductance Calculations. New York: Dover Publications, 1973 , pp. 55-58.

[8] W. M. McKeeman, "Adaptive numerical integration by Simpson's rule, Algorithm 145," Commun. ACM, vol. 5, no. 12, pp. 604-605, 1962.

[9] A. E. Ruehli, "Inductance calculations in a complex integrated circuit environment," IBM J. Res. Develop., vol. 22, no. 3, pp. 470-481, 1972.

[10] M. Enohnyaket and J. Ekman, "Analysis of air-core reactors from DC to very high frequencies using PEEC models," IEEE Trans. Power Delivery, accepted for publication.

[11] A. Müsing, C. Zingerli, and J. W. Kolar, "PEEC-based numerical optimization of compact radial position sensors for active magnetic bearings," in Proc. 5th CIPS Conf., 2008, pp. 403-408.

[12] G. Antonini, J. Ekman, A. C. Scogna, and A. E. Ruehli, "A comparative study of PEEC circuit elements computation," in Proc. IEEE Int. Symp. Electromagn. Comp., 2, 2003, pp. 810-813.

[13] G. A. Campbell, "Mutual inductances of circuits composed of straight wires," Phys. Rev., vol. 5, pp. 452-458, Jun. 1915. 